\title{
HISTERECTOMÍA Y ANSIEDAD: ESTUDIO COMPARATIVO ENTRE DOS TIPOS DE PREPARACIÓN PSICOLÓGICA PREQUIRÚRGICA*
}

\section{Hysterectomy and anxiety: comparison between two methods of preoperative psychological preparation} Andrea Cárdenas**, Cynthia Quiroga**, Martha Restrepo***, Daniel Cortés, M.D.****

Recibido: mayo 11/05-Revisado: junio 24/05-Aceptado: agosto 12/05

\section{RESUMEN}

La histerectomía es valorada por las pacientes como una situación altamente estresante, que genera fuertes reacciones emocionales con reconocido efecto negativo sobre la recuperación posquirúrgica. Para contrarrestar dichos efectos psicológicos adversos se han desarrollado diferentes tipos de programas de preparación para cirugía, los cuales buscan hacer más controlable y predecible el proceso pre y posoperatorio, facilitando la recuperación. El presente estudio buscó comparar dos tipos de preparación para cirugía en mujeres programadas para histerectomía abdominal en la Clínica Carlos Lleras Restrepo de Bogotá. El primer grupo de 30 mujeres, recibió una preparación basada en la consideración particular de las estrategias de afrontamiento. El segundo grupo, también de 30 mujeres, recibió preparación mediante información escrita. Como variables de resultado se estudiaron los niveles de ansiedad pre y posoperatoria, la magnitud del dolor postoperatorio percibido y la presencia de complicaciones posquirúrgicas inmediatas. Los resultados revelaron que las participantes del grupo que reci-

* Trabajo galardonado con la mención meritoria otorgada por la Facultad de Ciencias Humanas de la Universidad Nacional de Colombia.

** Psicólogas, Universidad Nacional de Colombia. Correo electrónico: acardenasj@unal.edu.co

*** Psicóloga, profesora asociada, Departamento de Psicología, Facultad de Ciencias Humanas, Universidad Nacional de Colombia.

**** Gineco-obstetra, Docente adscrito Departamento de Gineco-obstetricia, Facultad de Medicina, Universidad Nacional de Colombia. bió el primer tipo de programa presentaron mayor disminución en la ansiedad $(p<0,01)$, menor nivel de dolor percibido $(p<0,05)$ y una baja frecuencia de complicaciones, que aquellas que recibieron la preparación a través de información escrita.

Palabras clave: histerectomía, psicología, ansiedad, dolor posoperatorio, complicaciones

\section{SUIMMARY}

Hysterectomy is perceived by patients as a highly stressing situation that generates strong emotions with well-known negative effects on postoperative recovery. In order to avoid those deleterious effects, different methods for preoperative psychological preparation have been developed, aiming for a more predictable and controlled postoperative period and an easier recovery. The present research aimed to compare the effects of two different kinds of psychological preparation programs on women undergoing hysterectomy at the Carlos Lleras Restrepo Clinic. The former group consisting of 30 women received a preparation program based upon their coping strategies. The second group, also consisting of 30 women, was provided with written information as the only method for preoperative psychological preparation. Preoperative anxiety levels, postoperative perceived pain and immediate postoperative complications were measured in both groups as endpoints. The results showed that patients in the first group had lower levels of anxiety $(p<0,01)$, 
less postoperative pain $(p<0,05)$ and a lower incidence of complications compared to those who were prepared just by providing written information.

Key words: hysterectomy, psychology, anxiety, postoperative pain, complications

\section{INTRODUCCIÓN}

La histerectomía es la segunda cirugía ginecológica más frecuente, después de la cesárea, según cifras provenientes de Norteamérica y Europa. Igual sucede en la Clínica Carlos Lleras de Bogotá, donde se realizó el presente estudio. ${ }^{1,2}$

La implementación de esta intervención quirúrgica mayor, está indicada como tratamiento en múltiples trastornos ginecológicos, benignos y neoplásicos, cuyas manifestaciones clínicas van en detrimento de la calidad de vida de las mujeres que las padecen. ${ }^{3,4}$ Dicho efecto negativo puede llegar a ser potenciado por las consecuencias psicológicas adversas propias de la histerectomía.

La resección del útero puede significar una pérdida parcial de aspectos significativos para la identificación como mujeres de las pacientes a quienes se les practica, pues aunque este órgano normalmente es invisible, se hace visible ante su ausencia, ${ }^{5}$ lo cual, como es de esperarse, tiene efectos emocionales que pueden incluir la vivencia como una pérdida afectiva, que provoca la iniciación de un proceso de duelo, modificaciones en la autoestima, en el estado de ánimo, en el deseo sexual y en las relaciones de pareja posteriores a la cirugía. ${ }^{5,6}$ Dichas respuestas obedecen, entre otras razones, al hecho de que en nuestra cultura el útero ha sido simbolizado como parte determinante de la identidad femenina debido al papel protagónico que desempeña en la procreación y la maternidad, funciones por las que históricamente la mujer ha sido definida y valorada en la sociedad.?

Se ha encontrado que entre la población femenina existe una serie de creencias y actitudes relacionadas con la histerectomía y sus consecuencias futuras. Entre los temores que se suelen mencionar frente a este procedimiento están la sensación de "quedar huecas", el aumento de peso, la pérdida del deseo sexual y las percepciones erróneas que sus compañeros puedan tener respecto a la histerectomía. ${ }^{8}$ El reconocimiento de las percepciones que tiene la mujer sobre esta intervención quirúrgica brinda una base rica para el desarrollo de intervenciones que mejoren el cuidado de su salud. ${ }^{9}$

La histerectomía puede ser valorada por las pacientes como un evento amenazante, lo cual puede generar altos niveles de ansiedad debidos a la ausencia de control por parte de la paciente y al carácter impredecible de la situación quirúrgica. ${ }^{10}$ Estas alteraciones en el estado de ánimo tienen efecto negativo sobre el proceso de recuperación postoperatoria, haciéndolo más lento y complicado. ${ }^{11,12}$ Siguiendo el modelo psiconeuroinmunológico, es posible afirmar que altos niveles de ansiedad pueden estar altamente correlacionados con alteraciones en el proceso de cicatrización, con una mayor percepción de dolor posquirúrgico, con baja adherencia a las recomendaciones terapéuticas, con la acción y los efectos secundarios de los medicamentos anestésicos y con presencia de complicaciones posquirúrgicas entre las que se incluyen infecciones, emésis y cefalea. ${ }^{13}$

Con la finalidad de facilitar la experiencia quirúrgica, reduciendo los efectos emocionales indeseables y propiciando, de manera indirecta, una más adecuada recuperación posquirúrgica, se han desarrollado una serie de programas de preparación psicológica prequirúrgica, que en un gran número han estado centrados en la provisión de información relacionada con la cirugía. ${ }^{14,15}$ Con mujeres programadas para histerectomía se ha implementado el uso de folletos informativos, cuyo efecto sobre los niveles de ansiedad ha variado en relación con el estilo de afrontamiento de cada mujer. Este último aspecto demuestra que los folletos informativos no son universalmente efectivos y que la decisión respecto a su uso debe ser tomada con base en la evaluación de las diferencias individuales respecto a los comportamientos y esfuerzos cognitivos utilizados para hacer frente a las situaciones estresantes. ${ }^{3}$ Lo conveniente es dar a cada paciente la cantidad de información que necesita. ${ }^{16}$ 
En diversos estudios, ${ }^{17,}{ }^{18}$ se documenta el desarrollo de protocolos de preparación para cirugía ginecológica en los cuales se combinan diferentes técnicas de intervención psicológica, encontrándose que las pacientes que se benefician de dichos programas han presentado una recuperación posoperatoria más rápida y adecuada que sus controles. Aunque estas intervenciones han demostrado su efectividad, han sido aplicadas, en su mayoría, de forma estandarizada, dejando de lado las diferencias individuales. Con el propósito de maximizar su efectividad los programas de preparación para cirugía deben ser cortos, inmediatamente efectivos y deben tener en consideración la variedad de esfuerzos de afrontamiento que el individuo emplea en esta situación. ${ }^{19}$

El objetivo de la presente investigación fue comprobar los efectos de un programa de preparación psicológica para cirugía basado en las estrategias de afrontamiento de cada una de las mujeres de un grupo programado para histerectomía abdominal total, y compararlos con los efectos de un programa de preparación basado en el suministro de información escrita. Igualmente se buscó comprobar las hipótesis según las cuales ambos programas tendrían efectos positivos sobre la recuperación posquirúrgica de las pacientes, aunque aquellas que recibieran el programa basado en sus estrategias de afrontamiento presentarían una mayor reducción en sus niveles de ansiedad pospreparación, menores niveles de dolor posquirúrgico y menor frecuencia de complicaciones posoperatorias (emésis, fiebre y taquicardia), que aquellas que recibieran el programa estándar basado en información.

\section{MATERIALES Y MÉTODOS}

Diseño: se realizó un ensayo clínico controlado ciego simple, en la Clínica Carlos Lleras Restrepo, de Bogotá, hospital general de atención de segundo nivel que atiende pacientes afiliados a la seguridad social ofrecida por la aseguradora estatal (ISS). Allí se realizan cada año un promedio de 450 histerectomías abdominales totales (HAT).
Población: participaron 60 mujeres, en edad fértil y perimenopáusicas, quienes en un $86 \%$ tenían diagnóstico de miomatosis uterina, razón por la cual estaban programadas para histerectomía abdominal total.

Procedimiento: mediante un procedimiento de asignación aleatoria simple usando una tabla de números aleatorios, se asignó a las participantes en uno de dos grupos, uno experimental $(n=30)$ que participaría en un programa de preparación para cirugía basado en sus estrategias de afrontamiento y uno de control $(n=30)$ que recibiría un programa estándar basado solamente en el suministro de información escrita.

Las participantes del grupo experimental recibieron un programa de preparación basado en el apareamiento de una matriz de Técnicas de Intervención Psicológica con los resultados obtenidos por cada paciente en la COSS (Coping mit Operativen Streb Skala). La fase de intervención, aplicada en una única sesión individual con una duración aproximada de 60 minutos, se dividía en tres componentes; uno de suministro de información verbal por demanda, relacionada con la cirugía y sus implicaciones físicas y emocionales; uno de implementación de técnicas cognitivas y de apoyo emocional, que se constituía en un espacio de expresión acerca de las emociones suscitadas por la cirugía y sus efectos en aspectos individuales y familiares; y en última instancia, uno de entrenamiento en habilidades de afrontamiento (búsqueda de apoyo social y pautas de auto cuidado posoperatorio) y de manejo del estrés (respiración profunda y/o imaginería). Al finalizar la sesión, a cada participante se le entregaba un ejemplar del folleto informativo "¿Podrías decirme algo sobre la histerectomía?”. Las participantes del grupo de control únicamente recibieron el folleto, en una sesión individual de 20 minutos, necesarios para la aplicación de los instrumentos de evaluación.

El folleto informativo es un instrumento de intervención, a través del cual se busca proporcionar información clara, sencilla y corta que facilite la 
comprensión del proceso patológico, quirúrgico e intrahospitalario, y de sus implicaciones biopsicosociales a corto y largo plazo. Además, incluye una sección en la que se plantean los principales mitos y tabúes alrededor de la histerectomía y su contraste con la realidad.

Variables a medir: como variables de resultado evaluadas en ambos grupos se utilizaron los niveles de ansiedad presentados en tres momentos del proceso quirúrgico (antes de la aplicación de la preparación psicológica, una hora después de haber finalizada dicha aplicación y 24 horas después de la histerectomía) medidos a través de la versión mexicana del Inventario de Ansiedad Rasgo-Estado IDARE; ${ }^{20}$ el nivel de dolor posoperatorio 24 horas después de la cirugía autorreportado por medio de una Escala Análoga Visual de Dolor (EAVD); y la presencia de algunas complicaciones posquirúrgicas inmediatas (fiebre, emésis y taquicardia), información tomada de la historia clínica y las hojas de enfermería de cada paciente y consignada en un formato prediseñado. Las estrategias de afrontamiento, que se emplearon como variable para la definición del tratamiento requerido por cada participante en el grupo experimental, fueron evaluadas con la Escala de Afrontamiento en Estrés Quirúrgico COSS (Coping mit Operativen Streb Skala), ${ }^{19}$ instrumento traducido y sometido a un proceso de validación de contenido a través de jueces expertos, cuyo resultado fue un índice de confiabilidad de $0,86 .{ }^{21}$ A través de un consentimiento informado todas las participantes dieron su autorización para la aplicación de los instrumentos de evaluación y el uso anónimo de la información obtenida.
Análisis: las variables psicológicas (auto reportes) fueron analizadas a través de la prueba t de Student para muestras independientes, con lo que se buscaba probar las hipótesis correspondientes por medio de la diferencia de medias. Las variables de recuperación fisiológica se presentan por medio de análisis de frecuencias. Se utilizó el programa SPSS 7.5 (Statistical Package for the Social Sciences) para Windows.

\section{RESUILTADOS}

\section{Características basales:}

La edad de las participantes osciló entre los 32 y los 58 años $(M=44,97$ años; $D E=5,028)$; en su mayoría pertenecían a los estratos socioeconómicos 2 y 3 (76,66\%) y un 53,33\% había alcanzado el nivel de educación básica secundaria, mientras un 25\% el de básica primaria. Las participantes tenían entre 0 y 9 hijos con un promedio de 2,45 ( $\mathrm{DE}=1.47)$ y en un $86,66 \%$ convivían con una pareja estable. El 80\% de las participantes había sido intervenido quirúrgicamente, al menos una vez, antes de la histerectomía.

En cuanto a la variable ansiedad (tabla 1), medida en tres momentos del proceso quirúrgico, se comprobó una diferencia significativa entre los dos grupos en la ansiedad previa a la cirugía. No obstante, en la segunda medición, no se encontraron diferencias significativas entre los grupos. Sin embargo, al realizar la prueba de diferencia de medias con los puntajes de ganancia (La diferencia entre las puntuaciones del pre-test y el post-test ${ }^{22}$ ) de ambos grupos se encontró que, como se había predicho, el grupo experimental mostró una mayor

Tabla 1. Prueba t de Student aplicada a las medias de ansiedad

\begin{tabular}{|l|c|c|c|c|c|}
\hline Variable & Media G.E. & Media G.C. & Significación (2 colas) & g.l. & t \\
\hline IDARE 1 & 40,73 & 36,13 & 0,018 & 58 & $2,42 * *$ \\
\hline IDARE 2 & 30,73 & 32,43 & 0,289 & 58 & $-1,069$ \\
\hline IDARE 3 & 28,26 & 30,73 & 0,161 & 58 & $-1,41$ \\
\hline Puntaje de ganancia entre grupos & 10 & 3,7 & 0,000 & 58 & $4,46 *$ \\
\hline
\end{tabular}

Nota. G.E. = Grupo Experimental; G.C. $=$ Grupo Control, g.l. $=$ grados de libertad

$* p<0,01, * * p<0,05$ 
reducción en sus niveles de ansiedad como efecto del tratamiento recibido, que el grupo de control. La tercera medición de ansiedad no mostró diferencias significativas entre los grupos. En la gráfica 1 se presenta la evolución de la ansiedad en ambos grupos a lo largo del proceso quirúrgico.

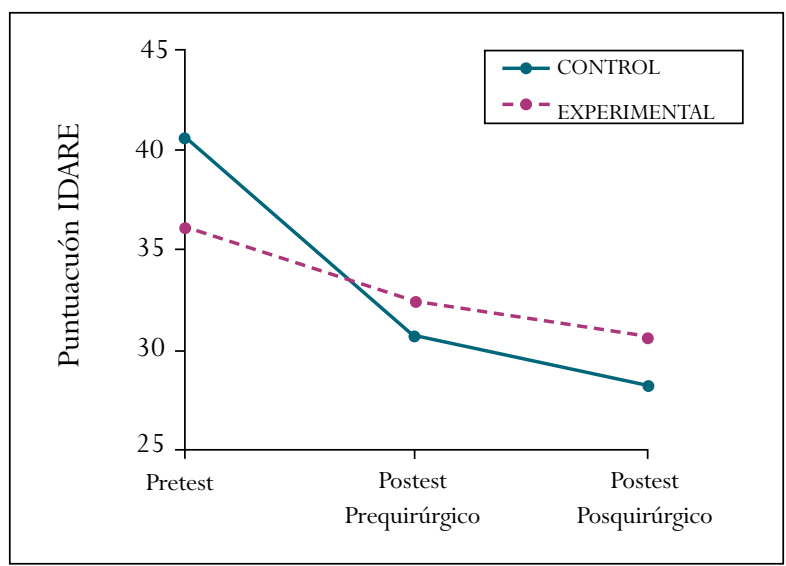

Gráfica 1. Evolución de los niveles de ansiedad a lo largo del proceso quirúrgico

Con relación al dolor autorreportado en la EAVD, se evidenció que el grupo experimental tuvo una media de 4,13 (DE $=2,47)$ y el grupo de control de 5,46 (DE=2,22), $t(58)=-2,19, p<$ 0,05 , lo cual demuestra que existe una diferencia estadísticamente significativa entre los puntajes de ambos grupos, siendo menor el nivel de dolor en el grupo experimental. Este hallazgo apoya la hipótesis según la cual, el grupo que recibiese el programa de preparación basado en estrategias de afrontamiento, reportaría menor dolor posquirúrgico, que aquel que solamente recibiese información.

Como se muestra en la gráfica 2, todos los índices evaluados presentaron mayor frecuencia en el grupo de control que en el experimental. Así, la frecuencia de complicaciones en ese grupo llegó a duplicar a aquella presentada por el grupo experimental, como fue el caso de las complicaciones catalogadas como "otros", entre las que se incluyen hematuria leve, retención de líquidos y marcada palidez facial, entre otros [grupo control 33,33\% $(n=10)$ grupo experimental 16,33\% $(n=5)]$. En el caso de la presencia de taquicardia la frecuencia en el grupo experimental $(21 \%, \mathrm{n}=7)$ fue casi triplicada por la del grupo control $(63,33 \%, n=19)$. Estos datos apoyan la hipótesis según la cual el grupo que recibiera el programa basado en estrategias de afrontamiento, tendría una más adecuada recuperación posoperatoria que quienes recibieran el programa basado en información.

\section{DISCUISIÓN}

Considerar las diferencias individuales en relación con las estrategias de afrontamiento como base para la implementación de un programa de preparación psicológica para histerectomía, nos permitió comprobar que este tipo de procedimiento facilita una más adecuada adaptación al proceso quirúrgico en términos de una mayor reducción en la ansiedad preoperatoria y de una adecuada recuperación posquirúrgica que incluye la percepción de bajos niveles de dolor. ${ }^{12,15,16}$

Los efectos positivos del programa sobre la ansiedad no se dieron solamente en relación con la percepción que las participantes tenían respecto a su propio estado de ánimo y a la situación quirúrgica, sino que además se vieron reflejados en los reportes de dolor posoperatorio. ${ }^{13}$ Así, aunque era de esperarse un aumento en el estado de ansiedad ante la inminencia de la cirugía, las mujeres que recibieron este programa estaban en capacidad de controlar su activación emocional y somática, influyendo indirectamente en su per-

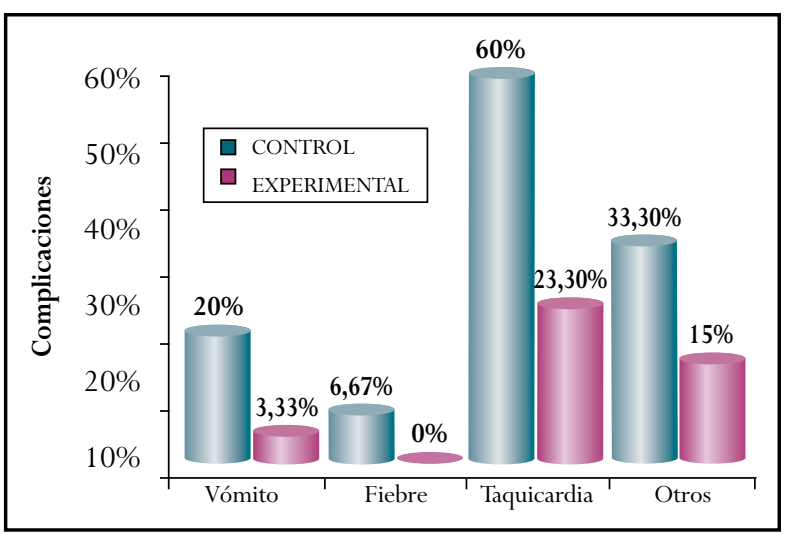

Gráfica 2. Presencia de complicaciones posquirúrgicas. 
cepción posterior de dolor. El autocontrol que estas mujeres estaban en capacidad de ejercer sobre su estado emocional, también influyó sobre la baja frecuencia de complicaciones posquirúrgicas. En este aspecto, se pudo establecer una relación entre los niveles de ansiedad, por una parte, y la acción y los efectos colaterales de los medicamentos anestésicos, por la otra. ${ }^{15}$ La baja frecuencia de complicaciones, del grupo experimental también pudo estar relacionada con los bajos niveles de dolor posquirúrgico percibido, pues en la respuesta ante el dolor se evidencian cambios neuroendocrinos, tales como la presencia de un incremento en los niveles de cortisol en el plasma sanguíneo, que ponen de manifiesto la reacción típica del sistema nervioso autónomo ante las situaciones de estrés. Entre los cambios fisiológicos originados a partir de esta respuesta se encuentran el aumento de la frecuencia cardiaca y la vasoconstricción cutánea; mismas reacciones que en este caso se vieron reducidas ante los bajos niveles de dolor percibido. ${ }^{23}$

$\mathrm{Al}$ diseñar este tipo de intervención psicológica es importante tener presente la multiplicidad de factores que intervienen en la percepción que las mujeres tienen respecto a la histerectomía y que pueden afectar directamente su vivencia de la situación quirúrgica. En consecuencia se hace indispensable la implementación de diversas técnicas psicológicas que tomen en consideración sus actitudes, creencias y respuestas emocionales, aspectos cuya relevancia no se tiene en cuenta en los programas que se limitan a proporcionar información.

\section{CONCLUSIONES}

De todo lo anterior es posible concluir que:

- Se hace necesario llevar a cabo programas de preparación para cirugía, en forma interdisciplinaria, como parte de la atención que deben recibir las pacientes ginecológicas, lo cual redunda en una mayor calidad asistencial y en una mejor adaptación física y psicológica posquirúrgica.
- Al desarrollar dichos programas se requiere tomar en cuenta las diferencias individuales en relación con las estrategias de afrontamiento y con las necesidades específicas referentes a la provisión de información, al apoyo psicológico y al entrenamiento en habilidades.

- El impacto de la implementación de un programa de preparación psicológica basado en las estrategias de afrontamiento sobre indicadores tales como ansiedad, dolor y complicaciones posquirúrgicas, tiene efectos más positivos sobre las pacientes programadas para histerectomía, en comparación con los efectos de un programa de preparación basado solamente en la provisión de información.

- Esta intervención es rentable ya que no demanda mayores recursos humanos, con un impacto muy favorable en la reducción de complicaciones posquirúrgicas que pueden generar costos hospitalarios.

\section{REFERENCIAS}

1. Cortés D, García G, Reyes L. Estadística de procedimientos quirúrgicos. Septiembre 15 a Octubre 31 de 2003. Universidad Nacional de Colombia. Clínica Carlos Lleras Restrepo; 2003.

2. Cortés D, Gómez G, Cediel E. Estadísticas de procedimientos quirúrgicos. Agosto 1 a Septiembre 31 de 2003. Universidad Nacional de Colombia. Clínica Carlos Lleras Restrepo; 2003.

3. Hunter M, Walker A. Gynecological Problems. En: Bellack, A.S.; Hersen, M. (Eds.). Comprehensive Clinical Psychology 1998. Elsevier version electrónica [Visitado 2004 May 08] http://www.ccp-online. com

4. Kinnick V, Lenners D. Impact of hysterectomies on women's lives: a prospective study. J Women Aging 1995;7:133-44.

5. Fernández M, Ospina B, Múnera A. La sexualidad en pacientes con cáncer de mama o cérvix sometidas a tratamiento quirúrgico en el Hospital General, Hospital San Vicente de Paúl e Instituto de Cancerología de la Clínica las Américas, Medellín; 2002.

6. Mingo C, Herman CJ, Jasperse M. Women's stories: ethnic variations in women's attitudes and experiences of 
menopause, hysterectomy, and hormone replacement therapy. J Womens Health Gend Based Med 2000;9 Suppl 2:S27-38.

7. González M. Subjetividad y ciclos vitales de las mujeres. Madrid: Siglo XXI; 1999.

8. Groff JY, Mullen PD, Byrd T, Shelton AJ, Lees E, Goode J. Decision making, beliefs, and attitudes toward hysterectomy: a focus group study with medically underserved women in Texas. J Womens Health Gend Based Med 2000;9 Suppl 2:S39-50.

9. Lewis CE, Groff JY, Herman CJ, Mckeown RE, Wilcox LS. Overview of women's decision making regarding elective hysterectomy, oophorectomy, and hormone replacement therapy. J Womens Health Gend Based Med 2000; 9 Suppl 2:S5-14.

10. Krohne HW. Vigilance and cognitive avoidance as concepts in coping research. En: Krohne HW, editor. Attention and avoidance. Strategies in coping with aversiveness. Seattle, Toronto: Hogrefe \& Huber; 1993. p. 19-50.

11. Munafo MR, Stevenson J. Anxiety and surgical recovery. Reinterpreting the literature. J Psychosom Res 2001;5:589-96.

12. Johnston M, Wallace L. (Eds.). Stress and medical procedures. Oxford, England: Oxford University Press; 1990.

13. Kiecolt-Glaser J, Page G, Marucha, PT, Maccallum RC, Glaser R. Psychological influences on surgical recovery. Perspectives from psychoneuroimmunology. Am Psychol 1998;53:1209-18.

14. Moix J, López E, Otero J, Quintana C, Ribera C, Saad I, Gil A. Eficacia de los folletos informativos para suministrar información acerca de la operación y la convalecencia. Calidad Asistencial 1995;5:280-86.
15. Mahler HI, Kulik JA. Effects of a videotaped information intervention for spouses on spouse distress recovery from surgery. Health Psychol 2002; 21 : 427-37.

16. Moix J. Disminución de la ansiedad como factor de mejora de la calidad asistencial en pacientes quirúrgicos. Calidad Asistencial 1998;13:160-5.

17. Callaghan P, Cheung L H. The effect of pre-operative psychological interventions on post-operative outcomes in Chinese women having an elective hysterectomy. $\mathrm{Br}$ Journal Health Psychol 2002;7:247-52.

18. Lookinland S, Pool M. Study on effect of methods of preoperative education in women. AORN J 1998;67:203-13.

19. Krohne HW, deBruin J, El-Giamal M, Schmukle SL. The assessment of surgery-related coping: the coping with surgical stress scale (COSS). Psychol Health 2000;15:135-49.

20. Spielberger C, Díaz-Guerrero R. IDARE. Inventario de ansiedad: rasgo-estado. Manual e instructivo. México: Manual Moderno; 1975.

21. Cárdenas A, Quiroga C. Efectos de un Programa de Preparación Psicológica para Cirugía basado en Afrontamiento vs. Un Programa Estándar basado en Información. Estudio Comparativo con Mujeres Programadas para Histerectomía Abdominal. Universidad Nacional de Colombia; 2004.

22. Hernández R, Fernández C, Baptista P. Metodología de la investigación (Research Metodology). México D.F.: McGrawHill Interamericana; 2003.

23. Valdés M, Flóres T. Psicobiologia del estrés.. Barcelona: Ed Martínez Roca; 1990.

Conflicto de intereses: El presente estudio es un trabajo investigativo y académico desarrollado bajo los lineamientos metodológicos y las políticas del Departamento de Psicología de la Universidad Nacional de Colombia, como parte del trabajo de grado de las psicólogas Andrea Cárdenas y Cynthia Quiroga. No recibió otra financiación más que la de los propios autores. 